\title{
A High-Rate Generalized Coded Delay Diversity Scheme and its Diversity-Multiplexing Tradeoff
}

\author{
Rahul Vaze \\ ECE Dept. \\ Indian Institute of Science \\ Bangalore -560012 INDIA \\ email: rahulv@protocol.ece.iisc.ernet.in
}

\author{
V Shashidhar \\ Beceem Communication Pvt. Ltd., \\ Bangalore -560012 INDIA \\ email: vshashidhar@beceem.com
}

\author{
B. Sundar Rajan \\ ECE Dept., \\ Indian Institute of Science \\ Bangalore -560012 INDIA \\ email: bsrajan@ece.iisc.ernet.in
}

\begin{abstract}
Multiple antennas can be used for increasing the amount of diversity (diversity gain) or increasing the data rate (the number of degrees of freedom or spatial multiplexing gain) in wireless communication. As quantified by Zheng and Tse [1], given a Multiple Input Multiple Output (MIMO) channel, both gains can, in fact, be simultaneously obtained, but there is a fundamental tradeoff (called the diversity-multiplexing tradeoff) between how much of each type of gain any coding scheme can extract. It is well known that Space-Time Trellis Codes (STTC) can be used to achieve full-diversity and better coding gain than Space-Time Block Codes (STBC). There have many STBC constructions [2]-[4], which achieve the diversitymultiplexing tradeoff, but to the best of our knowledge no such construction is proposed for the case of STTC. Delay diversity scheme used to construct STTC introduced in [5] is known to achieve full-diversity, for any number of transmit antennas. In this paper, we show that the delay diversity scheme can achieve the optimal diversity-multiplexing tradeoff, only for one receive antenna. Then we propose a generalized construction of a highrate generalized coded delay diversity scheme (HRGCDD). We show that HRGCDD scheme meets both the extreme points (corresponding to zero diversity gain and zero multiplexing gain) of the optimal diversity-multiplexing tradeoff curve for any number of transmit and receive antennas. Also by using HRGCDD scheme we construct STTC for 2 transmit antennas. Furthermore we also show that the new proposed STTC achieves the optimal diversity-multiplexing tradeoff for 2 transmit and 2 receive antennas by simulation.
\end{abstract}

\section{INTRODUCTION}

Space-Time Trellis Codes (STTC) have been introduced in [5] to provide improved error performance for wireless systems using multiple transmit antennas. It has been shown [5] that such codes constructed by delay diversity scheme can provide, full diversity gain as well as additional signal-to-noise ratio (SNR) advantage, i.e. coding gain, but with some restrictions on the number of the states of the trellis. A general method to construct STTCs for any number of states, which ensures fulldiversity and good coding gain is given by [6], where coding in conjunction with delay diversity is used.

It is well known, that at high SNRs the capacity of Rayleigh fading channel with $n_{t}$ transmit and $n_{r}$ receive antennas is

$$
C\left(n_{t}, n_{r}, \mathrm{SNR}\right)=\min \left\{n_{t}, n_{r}\right\} \log \mathrm{SNR}+O(1)
$$

${ }^{1}$ This work was partly supported by the DRDO-IISc program on Advanced Research in Mathematical Engineering and by the Council of Scientific and Industrial Research (CSIR), India, through Research Grant (22(0365)/04/EMRII) to B.S.Rajan. where $O(1)$ is a constant. The above expression shows that the achievable data rate increases with SNR as $\min \left\{n_{t}, n_{r}\right\} \log$ SNR. This advantage due to multiple antennas is called the spatial multiplexing gain.

Though most of the research in designing good STTCs has been in obtaining full-diversity codes and maximizing the coding gain, no effort seems to have been made to construct STTCs with maximum spatial multiplexing.

The contributions of this paper are:

- We show that the existing delay diversity schemes do not achieve the optimal diversity-multiplexing tradeoff curve for more than one receive antenna.

- We propose a high-rate generalized coded delay diversity (HRGCDD) scheme to construct STTCs, which cannot be viewed as generalized coded delay diversity scheme [6].

- The HRGCDD scheme achieves both the extreme points of the optimal diversity-multiplexing tradeoff curve for any number of transmit and receive antennas.

- We show by simulation, that STTC constructed for 2 transmit antennas from HRGCDD scheme achieves the optimal diversity-multiplexing tradeoff for both 1 and 2 receive antennas.

The rest of the paper is organized as follows. Section II presents the system model. In Section III, we discuss the existing schemes to construct STTCs and study their diversitymultiplexing tradeoffs. We introduce the HRGCDD scheme which achieve both the extreme points on the diversitymultiplexing tradeoff and construct STTC for 2 transmit antennas from the HRGCDD scheme in Section IV. For 2 transmit antennas, we compare the performance of the existing schemes viz a viz our new proposed HRGCDD scheme by simulation in Section V with 1 and 2 receive antennas. Section VI contains the concluding remarks.

\section{System Model AND Design CRiteria}

The system model we consider is a MIMO wireless communication system with $n_{t}$ transmit antennas and $n_{r}$ receive antennas. A Space-Time Trellis Code (STTC) for such a channel model is a finite set $\mathcal{C}$ of $n_{t} \times L$ matrices (called codewords) where $L$ is an integer, such that the channel coefficients do not change for $L$ symbol intervals. At each time $t$, depending on the state of the encoder and the input bits, a transition branch is chosen. If the label of this branch is $\mathbf{x}_{\mathbf{t}}=\left(x_{t}^{1}, x_{t}^{2}, \ldots, x_{t}^{n_{t}}\right)^{T}$, 
then transmit antenna $i$ is used to transmit constellation symbol $x_{t}^{i}$ and all these transmissions are simultaneous. An element $\mathbf{X} \in \mathcal{C}$, is of the form $\mathbf{X}=\left(\mathbf{x}_{1}, \mathbf{x}_{\mathbf{2}}, \ldots, \mathbf{x}_{\mathbf{t}}, \ldots, \mathbf{x}_{\mathbf{L}}\right)$.

If $\mathbf{X} \in \mathcal{C}$ is the transmitted codeword and under the assumption that $\mathcal{E}\left[\operatorname{tr}\left(\mathbf{X X}^{*}\right)\right]=n_{t} L$ and the average signalto-noise (SNR) at each receive antenna is SNR, the received signal is

$$
\mathbf{Y}=\sqrt{\frac{\mathrm{SNR}}{n_{t}}} \mathbf{H X}+\mathbf{W}
$$

where $\mathbf{Y} \in \mathbb{C}^{n_{r} \times L}, \mathbf{H} \in \mathbb{C}^{n_{r} \times n_{t}}$ is the channel matrix and $\mathbf{W} \in \mathbb{C}^{n_{r} \times L}$ is the additive noise, both with entries independent complex Gaussian with zero mean and unit variance. At high SNRs, the probability that the received matrix $\mathbf{Y}$ is decoded to a codeword matrix $\mathbf{X}^{\prime} \neq \mathbf{X}$ is $P\left(\mathbf{X} \rightarrow \mathbf{X}^{\prime}\right)=$ $\left(\prod_{i=1}^{\Lambda} \lambda_{i}^{2}\right)^{-n_{r}}$ SNR $^{-n_{r} \Lambda}$ where $\lambda_{1}, \lambda_{2}, \ldots, \lambda_{\Lambda}$ are the $\Lambda$ nonzero singular values of $\Delta=\mathbf{X}-\mathbf{X}^{\prime}$. The gain due to spatial diversity provided by $n_{t}$ transmit and $n_{r}$ receive antennas is called the diversity gain of the code and is equal to the negative of the exponent of SNR in the above expression. So, if $n_{t}$ and $n_{r}$ are the number of transmit and receive antennas, then the diversity gain can be at most equal to $n_{t} n_{r}$. Thus, in order to minimize the error probability, we should design a code $\mathcal{C}$ such that the difference matrix of any two codewords have full rank $\left(=n_{t}\right)$. Any STTC with such property is called a fullrank STTC. However, this design criteria is derived under the assumption that the data rate is fixed for all SNRs.

\section{A. Diversity-Multiplexing Tradeoff}

We will first give some necessary definitions and then briefly discuss the optimal tradeoff curve achievable for any scheme, whereby a scheme is a family of codes, one for each SNR level [1]. Let $\mathcal{C}(\mathrm{SNR})$ be the code corresponding to the SNR level SNR and the data rate in bits per channel use achieved by $\mathcal{C}(\mathrm{SNR})$ be $R(\mathrm{SNR})$.

Definition 1 ( [1]): A scheme $\{\mathcal{C}(\mathrm{SNR})\}$ is said to achieve a spatial multiplexing gain $r$ and diversity gain $d$ if

$$
\lim _{\mathrm{SNR} \rightarrow \infty} \frac{R(\mathrm{SNR})}{\log \mathrm{SNR}}=r \quad \text { and } \quad \lim _{\mathrm{SNR} \rightarrow \infty} \frac{\log P_{e}}{\log \mathrm{SNR}}=-d
$$

where $P_{e}$ is the average error probability.

Following the notation of [1], we use $\doteq$ for exponential equality. Thus, $2^{R(\mathrm{SNR})} \doteq \mathrm{SNR}^{r}$ and $P_{e} \doteq \mathrm{SNR}^{-d}$. Similarly, we use $\dot{\geq}, \dot{\leq}$ for exponential inequalities.

Though a scheme means a family of codes, one for each SNR level, a simple way of describing a scheme is to give a design (defined below) and a class of signal sets.

Definition 2 ([3]): A rate- $k / L, n_{t} \times L$ design over a subfield $K$ of the complex field $\mathbb{C}$, is a $n_{t} \times L$ matrix $\mathbf{M}\left(x_{1}, x_{2}, \ldots, x_{k}\right)$ with entries that are $K$-linear combinations of $k$ variables and their complex conjugates. We call $\mathbf{M}$ a full-rank design over a field $F \subset \mathbb{C}$ if every $\mathbf{M}\left(x_{1}, x_{2}, \ldots, x_{k}\right) \mid x_{i} \in F, i=1,2, \ldots, k$, is full-rank.

Zheng and Tse in [1] have obtained lower and upper bound on the optimal diversity-multiplexing tradeoff curve. For the case $L \geq n_{t}+n_{r}-1$, the optimal diversity-multiplexing tradeoff is given by $d(r)=d_{\text {out }}(r)=\left(n_{t}-r\right)\left(n_{r}-r\right)$.
If a scheme is described by a full-rank design over a field $F$ and a class of signal sets which are subsets of $F$, then the maximum diversity gain achieved by such a scheme is the diversity gain of any code in the scheme. Thus, a scheme which is a family of full-rank codes achieves the point $\left(0, n_{t} n_{r}\right)$ of the optimal diversity-multiplexing tradeoff. Schemes like VBLAST [7] achieve the point $\left(\min \left\{n_{t}, n_{r}\right\}, 0\right)$ of the optimal tradeoff curve.

\section{B. Capacity of Designs}

Let $\mathbf{X}$ be a rate- $k / L, n_{t} \times L$ design with $a_{1}, a_{2}, \ldots, a_{k}$ as the variables. If $\mathbf{X}$ is a design used to describe a STTC, then the received matrix is $\mathbf{Y}$ as in (1). Suppose, we can rewrite (1) as

$$
\tilde{\mathbf{y}}=\sqrt{\frac{\mathrm{SNR}}{n_{t}}} \widehat{\mathbf{H}} \tilde{\mathbf{a}}+\tilde{\mathbf{w}}
$$

where $\tilde{\mathbf{a}}$ is a $k \times 1$ vector containing either $a_{i}$ or $a_{i}^{*}$ but not both $a_{i}$ and $a_{i}^{*}$. Then from [8], we define the capacity of the design $\mathbf{X}$ as the capacity of the equivalent channel $\widehat{\mathbf{H}}$ given by

$$
C_{\mathbf{X}}\left(n_{t}, n_{r}, \mathrm{SNR}, \mathbf{H}\right)=\frac{1}{L} \log _{2}\left(\operatorname{det}\left(I_{n_{r} L}+\frac{\mathrm{SNR}}{n_{t}} \widehat{\mathbf{H}} \widehat{\mathbf{H}}^{\dagger}\right)\right)
$$

Clearly, $C_{\mathbf{X}}\left(n_{t}, n_{r}, \mathrm{SNR}\right)=\mathcal{E}_{\mathbf{H}}\left[C_{\mathbf{X}}\left(n_{t}, n_{r}, \mathrm{SNR}, \mathbf{H}\right)\right] \leq$ $C\left(n_{t}, n_{r}, \mathrm{SNR}\right)$. From [8], for a design to achieve capacity, it is necessary but not sufficient that $k \geq L \times \min \left\{n_{t}, n_{r}\right\}$. Also the design $\mathbf{X}$, can achieve the point $\left(\min \left\{n_{t}, n_{r}\right\}, 0\right)$ of the diversity-multiplexing tradeoff curve, only if the $\operatorname{rank}(\widehat{\mathbf{H}}) \geq$ $L \times \min \left\{n_{t}, n_{r}\right\}$.

\section{EXISTING FULL-RANK STTCS}

In [6], a general technique to construct full-rank STTC (under some conditions) by using coding in conjunction with the delay diversity scheme is presented to get better performance in terms of coding gain. Fig. 1 shows the coded delay diversity scheme for $n_{t}$ transmit antennas which achieves diversity $n_{t}$. For every channel use, $k$ bits enter the rate $k / n$ block encoder. The $n$ output bits of the block encoder are mapped by the

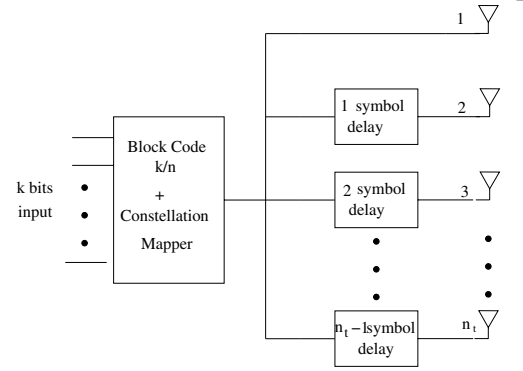

Fig. 1. Coded Delay Diversity Scheme for $n_{t}$ transmit antennas constellation mapper. The size of the signal constellation $\mathcal{S}$ is $2^{n}$. The constellation mapper, outputs one of the $2^{n}$ symbols from $\mathcal{S}$ for every $k$ bit input. The output of the constellation mapper is transmitted from the first transmit antenna. For the $i^{\text {th }}$ transmit antenna, the output of the constellation mapper is transmitted after $i-1$ channel uses for $i>1$. If $k=n$, then we get the delay diversity scheme of [5].

Fig. 2 is an example of full-rank STTC for 2 transmit antennas constructed by delay diversity scheme introduced in 


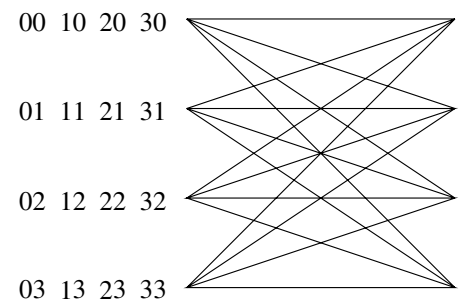

Fig. 2. 2 Tx, 4-PSK, $2 \mathrm{bps} / \mathrm{Hz}, 4$ state STTC

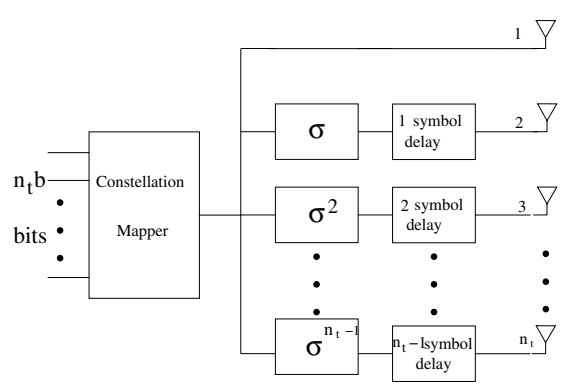

Fig. 3. High-Rate Generalized Coded Delay Diversity Scheme for $n_{t}$ transmit antennas

over the field $F=\mathbb{Q}\left(\omega_{n}, \omega_{m}\right)$, where $m=\operatorname{lcm}(n, 4)$ and $\sigma$ be a map from $F[\delta] \mapsto F[\delta]$ given by $\sigma(\delta)=\delta \omega_{n}$ and $\sigma$ restricted to $F$ is identity map. Consider the design $\mathbf{X}$ in equation (4) (top of next page), where $a_{1}, s_{j, i} \in \mathcal{S}, \forall i=0,1, \ldots, n_{t}-1$ and $j=0,1, \ldots, L-n_{t}-1$, then rank of the matrix $\mathbf{X}$ is $n_{t}$. We call this scheme as high-rate generalized coded delay diversity (HRGCDD) scheme.

Proof: From the design criteria [5], to prove that HRGCDD scheme is full-rank, we have to show that, if $c$ and $e$ are two distinct codewords of HRGCDD, then the difference matrix $c-e$ is full rank. Let's say that the two codewords $c$ and $e$ (where $c \neq e$ ), diverge at time $t_{d}$ for the first time, then the corresponding codeword difference matrix for the codewords $c$ and $e$ for the generalized coded delay diversity scheme is given by equation (5) (top of next page), where $\Delta x_{j, i}=c_{j, i}-e_{j, i}$, $\sigma^{i}$ denotes composition of $\sigma, i$ times and $*$ is any value from $F[\delta]$.

From the structure of the difference matrix it is clear that, to have the difference matrix full rank (i.e. rank $n$ ), we only need to show that $\sigma^{k}\left(\sum_{i=0}^{n-1} \Delta x_{t_{d}-1, i} \delta^{i}\right)=0$ if and only if $\Delta x_{t_{d}-1, i}=0, \forall i=0,1,2, \ldots, n$ and $k=0,1,2, \ldots, n$. To prove this, it is sufficient to prove that $1, \delta, \delta^{2}, \ldots, \delta^{n-1}$ form a basis of $F(\delta) / F$ and $\sigma$ is an automorphism of $F(\delta)$. Since $x^{n}-\omega_{m}$ is the minimal polynomial of $\delta$ over $F=\mathbb{Q}\left(\omega_{n}, \omega_{m}\right)$, $1, \delta, \delta^{2}, \ldots, \delta^{n-1}$ do not satisfy any linear equation in $n$ variables over $F$. Also, clearly $1, \delta, \delta^{2}, \ldots, \delta^{n-1}$ span the field $F[\delta]$ over $F$. Thus, $1, \delta, \delta^{2}, \ldots, \delta^{n-1}$ form a basis of $F[\delta]$ over $F$. Since $\sigma$ is a field homomorphism and $\operatorname{ker} \sigma \neq F[\delta]$ (which implies $\operatorname{ker} \sigma=0), \sigma$ is an automorphism. Thus, the HRGCDD scheme is full-rank.

Example 1: Let $n=n_{t}=2$ in Theorem 1 and $\mathcal{S}$ be a $Q A M$ signal set. Then we take $F=\mathbb{Q}\left(\omega_{2}, \omega_{4}\right)=\mathbb{Q}(j)$ and $x^{2}-j$ is irreducible over $F$. The automorphism $\sigma$ is given by $\sigma: \sqrt{j} \rightarrow-\sqrt{j}$. Then we have a full rank STTC for 2 transmit antennas given by Fig. 4 .

Though a scheme means a family of codes one for each SNR level, a simple way of describing a scheme is to give a design and a class of signal sets. Therefore varying the signal set, we get a family of codes, from which the variables of the design take value from. For example, for 2 transmit antennas by allowing the variables $x_{i j}$ in design (4) to take value from BPSK constellation, we get the code given in Fig.4. For increasing the data rate, we can use the same design with a bigger sized signal set and hence we get a family of codes one for each SNR level. For example using a 16 state STTC, 


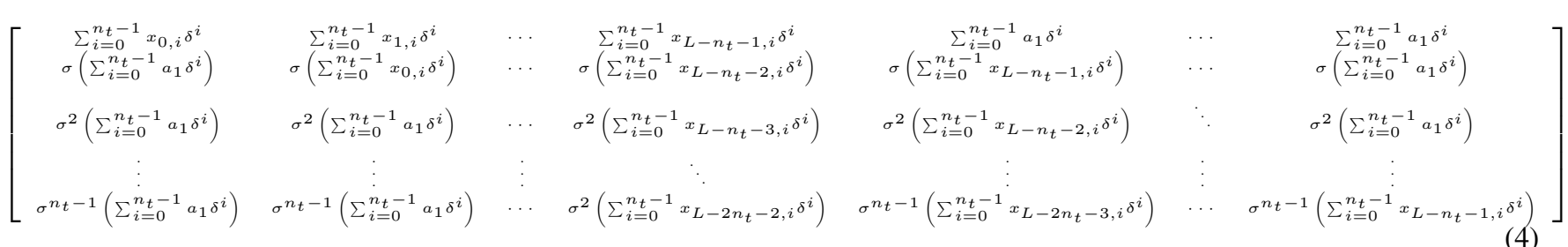

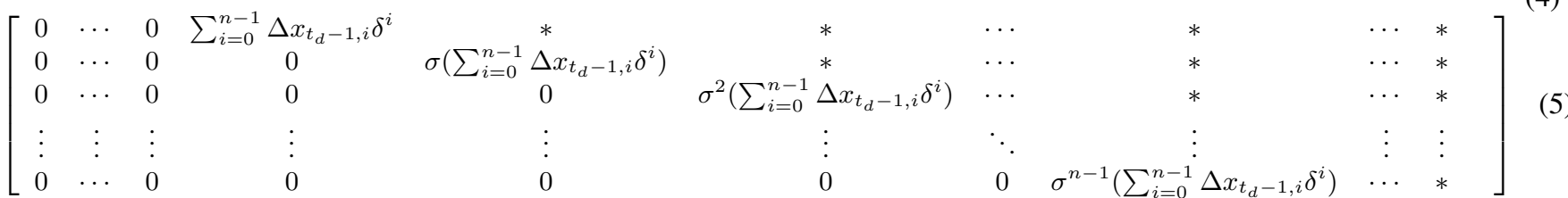

and the above design with 4 QAM as signal constellation we can get HRGCDD code for rate $4 \mathrm{bps} / \mathrm{Hz}$.

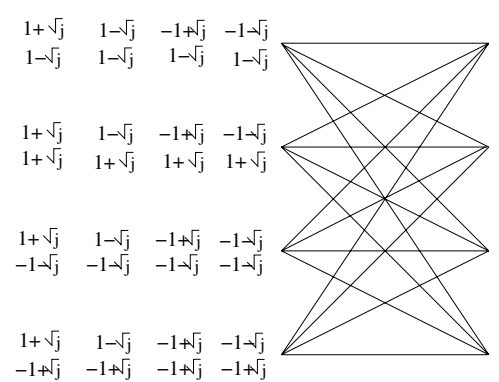

Fig. 4. New Code for $2 \mathrm{Tx}$, BPSK, 2bps/Hz, 4 state STTC

Theorem 2: The scheme $\mathcal{C}(\mathrm{SNR})$ obtained by HRGCDD, achieves the point $\left(0, n_{t} n_{r}\right)$ and $\left(\min \left\{n_{t}, n_{r}\right\}, 0\right)$ of the diversity-multiplexing tradeoff curve for $n_{r}$ receive antennas. Proof: Since $\mathcal{C}(\mathrm{SNR})$ is a full-rank code, the point $\left(0, n_{t} n_{r}\right)$ is achieved on the diversity-multiplexing tradeoff curve for $n_{t}$ transmit and $n_{r}$ receive antennas. From the HRGCDD scheme for $n_{t}$ transmit antennas, clearly, the entries $\mathbf{X}_{p, q}$ and $\mathbf{X}_{p^{\prime}, q^{\prime}}$ of design $\mathbf{X}$ are statistically independent except when $p^{\prime}=$ $p+i$ and $q^{\prime}=q+i$ for some $i=1,2, \ldots, n_{t}-1$. For $q=$ $1,2, \ldots, n_{t}$

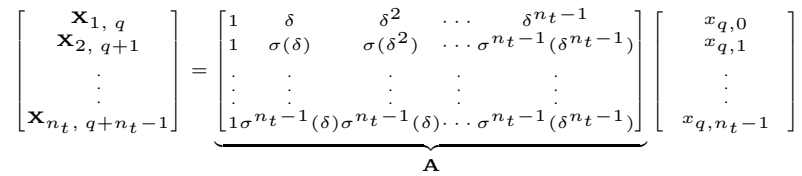

and let $A A^{H}=B=\left\{b_{i, j}, i, j=1,2, \ldots n_{t}\right\}$, then $b_{i, j}=$ $\sum_{k=0}^{n_{t}-1} \sigma^{i}\left(\delta^{k}\right)\left(\sigma^{j}\left(\delta^{k}\right)\right)^{*}=\sum_{k=0}^{n_{t}-1} \omega_{n_{t}}^{i k} \omega_{n_{t}}^{j k}=\sum_{k=0}^{n_{t}-1} \omega_{n_{t}}^{(i-j) k}$. But, $\sum_{k=0}^{n_{t}-1} \omega_{n_{t}}^{(i-j) k}=0$ if and only if $i \neq j$, otherwise equal to $n_{t}$. Therefore matrix $B=A A^{H}=n_{t} I_{n_{t}}$ and thus the two entries are statistically independent if $(p, q) \neq\left(p^{\prime}, q^{\prime}\right)$. Hence, we assume that our codeword matrix is equal to ã as given in equivalent channel model (2), and each entry of $\mathbf{x}$ is from a signal set $\mathcal{S}^{\prime}$ of the form $\left\{\sum_{i=0}^{n_{t}-1} x_{i} \delta^{j} \omega^{j k} \mid x_{i} \in \mathcal{S}\right\}$.

We now use successive nulling and canceling detection of $\mathrm{V}$ BLAST [7] and derive an upper bound on the error probability. With successive nulling and canceling process, the channel model is equivalent to $y_{i}=\sqrt{\frac{\mathrm{SNR}}{n}} g_{i} x_{i}+w_{i}$ where $g_{i}^{2}$ is the $i$-th decorrelator SNR gain which is a chi-squared distributed random variable with $2 i$ degrees of freedom. From [1], the pair-wise error probability for $i$-th decorrelator is

$$
P\left(x_{i} \rightarrow x_{i}^{\prime}\right)=P\left(g_{i}^{2}<\frac{n}{\mathrm{SNR}\left\|x_{i}-x_{i}^{\prime}\right\|^{2}}\right)
$$

Since the matrix $A A^{H}$ is unitary, the minimum squared Euclidean distance (MSED) of the set $S_{x}$ of vectors $\mathrm{x}$ is equal to the MSED of the SNR ${ }^{r / n_{t}}$ QAM signal set. Scaling the signal set such that the average power per entry of the codeword matrix $\mathbf{X}$ is equal to one, we have the MSED of $\mathrm{SNR}^{r / n_{t}}$ QAM signal set equal to SNR ${ }^{-r / n_{t}}$. Thus, (6) becomes

$$
P\left(x_{i} \rightarrow x_{i}^{\prime}\right) \doteq P\left(g_{i}^{2}<\frac{n}{\mathrm{SNR}^{1-r / n_{t}}}\right) \doteq \mathrm{SNR}^{-i\left(1-r / n_{t}\right)}
$$

Since, the number of neighbours for any QAM is at most equal to 4 , we have the error probability for $i$-th decorrelator as

$$
P_{e}^{(i)} \doteq \mathrm{SNR}^{-i\left(1-r / n_{t}\right)}
$$

Since, $P_{e}^{(1)} \leq P_{e}^{\prime}(\mathrm{SNR}) \leq \sum_{i} P_{e}^{(i)}$, where $P_{e}^{\prime}$ is the error probability with successive nulling and canceling detection, the error probability $P_{e}(\mathrm{SNR})$ with $\mathrm{ML}$ detection is given by

$$
P_{e}(\mathrm{SNR})<P_{e}^{\prime}(\mathrm{SNR}) \doteq \mathrm{SNR}^{-\left(1-r / n_{t}\right)}
$$

and thus $d_{H R G C D D}(r) \geq 1-r / n_{t}$. Hence the HRGCDD scheme achieve the points $\left(0, n_{t} n_{r}\right)$ and $\left(\min \left\{n_{t}, n_{r}\right\}, 0\right)$ of the diversity-multiplexing tradeoff curve for $n_{t}$ transmit and $n_{r}$ receive antennas. Thus the HRGCDD codes achieve the maximal achievable spatial multiplexing gain which is $n_{t}$, and also can exploit the maximum possible diversity gain.

\section{Simulation Results:}

In this section, we provide simulation results for the STTC constructed from HRGCDD scheme for 2 transmit antennas and compare its frame error rate performance with the existing scheme for 1 and 2 receive antennas. For 2 transmit antennas, Fig. 5, 6, 7 and 8 shows the frame error probability curves for various data rates for HRGCDD scheme and the existing delay diversity scheme for 1 and 2 receive antennas. For 1 receive antenna case, at high SNRs the gap between two adjacent curves for the HRGCDD scheme and the delay diversity schme, with data rates differing by 2 bits per channel use, is converging to $6 \mathrm{~dB}$. This indicates that at $d=0$, the data rate grows with SNR as $R=\log$ SNR for both the cases. Thus, the point $(1,0)$ of the trade-off curve is achieved by both the schemes for 


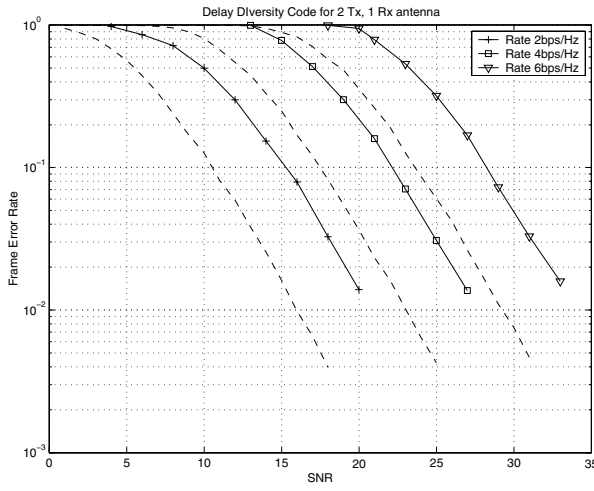

Fig. 5. Frame error probability curves (solid) for 4, 16, 64 QAM respectively and outage probability curves (dashed).

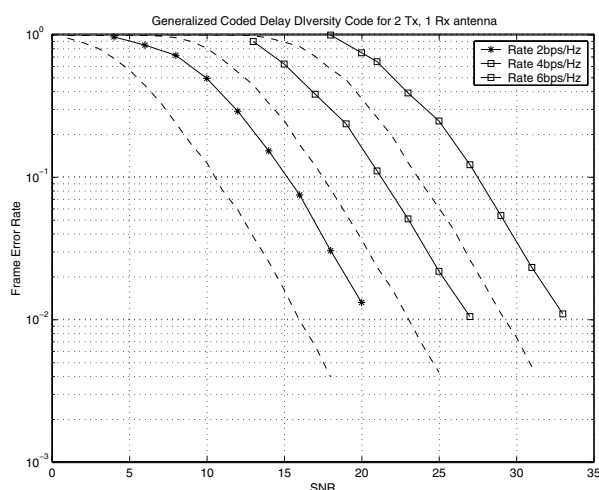

Fig. 6. Frame error probability curves (solid) for 4, 16, 64 QAM respectively and outage probability curves (dashed).

2 transmit and 1 receive antennas. For the 2 receive antenna case, the gap between two adjacent curves for the HRGCDD scheme is converging to $3 \mathrm{~dB}$ while for the delay diversity case it converges to $6 \mathrm{~dB}$ for the data rates differing by 2 bits per channel use. This indicates that at $d=0$, the data rate grows with SNR as $R=2 \log$ SNR for the HRGCDD scheme while it is $R=\log$ SNR for the delay diversity scheme. Also from the simulation plots, it can be seen that the frame error rate curves for 1 receive antenna converge towards the outage probability at high SNRs for both the codes, while for 2 receive antennas, only the curves for the HRGCDD scheme converge towards the outage probability at high SNRs. Therefore, both the delay diversity scheme and the HRGCDD scheme achieve the diversity-multiplexing tradeoff for 1 receive antenna but for 2 receive antennas only the HRGCDD scheme achieves the optimal diversity-multiplexing tradeoff.

\section{DISCUSSION:}

In this paper, we first show that the existing schemes to construct STTCs do not achieve the optimal diversity-multiplexing tradeoff, for $n$ transmit and $n$ receive antennas $(n \geq 2)$. We then give a new scheme called the high-rate generalized coded delay diversity (HRGCDD) scheme to construct full rank STTCs, which achieves both the extreme points (corresponding to zero diversity gain and zero multiplexing gain) of the optimal diversity-multiplexing tradeoff, while the existing schemes achieve only the point corresponding to zero multiplexing gain. For 2 transmit antennas case, we show by simulation,

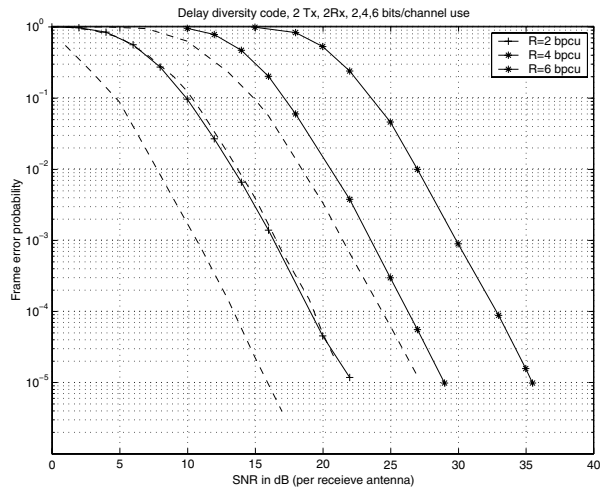

Fig. 7. Frame error probability curves (solid) for 4, 16, 64 QAM respectively and outage probability curves (dashed).

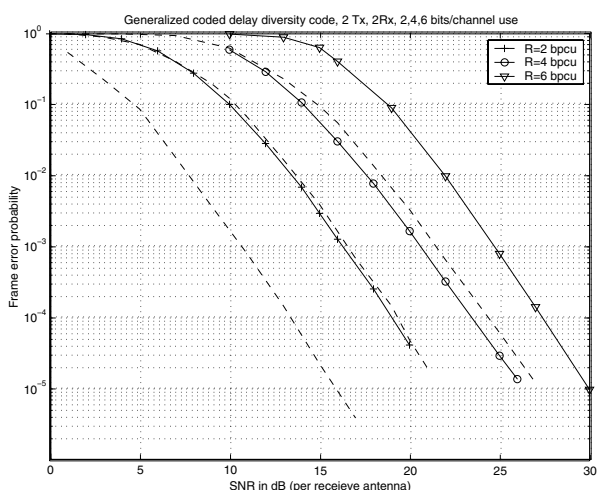

Fig. 8. Frame error probability curves (solid) for BPSK, 4 and 8 QAM respectively and outage probability curves (dashed).

that the delay diversity scheme achieves the optimal diversitymultiplexing tradeoff only for 1 receive antenna whereas the HRGCDD scheme achieves the optimal diversity-multiplexing tradeoff for both 1 and 2 receive antennas.

\section{REFERENCES}

[1] Lizhong Zheng and David N. C. Tse, " Diversity and Multiplexing: A Fundamental Tradeoff in Multiple-Antenna Channels," IEEE Trans.Inform. Theory, vol.49, no.5, pp.1073-1096, May 2003.

[2] Huan Yao and Gregory Wornell, "Structured space-time block codes with optimal diversity-multiplexing tradeoff and minimum delay," Proc. GLOBECOM 2003, Communication THeory Symposium, San Fransisco, Dec. $1-5,2003$, pp. 1941-1945.

[3] V. Shashidhar, B. Sundar Rajan and B.A.Sethuraman "STBCs using capacity achieving designs from crossed-product division algebras," Proceedings of IEEE ICC 2004, 20-24 June 2004, Paris, France, pp.827831.

[4] Jean-Claude Belfiore, Ghaya Rekaya and Emanuel Viterbo, "The Golden Code: A $2 \times 2$ Full-Rate Space-Time Code with Non-Vanishing Determinants, "Proceedings of IEEE International Symposium on Information Theory (ISIT 2004), Chicago, June 27- July 2, 2004 pp. 308.

[5] Vahid Tarokh,N.Seshadri and A. R. Calderbank, "Space-Time block codes for high data rate wireless communication:Performance criterion and code construction," IEEE Trans.Inform. Theory, vol.44, pp.744-765, Mar 1998. in Proc. IEEE ICC, v 3, 2002,pp. 1589-1595.

[6] T. Ananta Narayanan and B. Sundar Rajan, "A General Construction of Space-Time Trellis Codes for PSK signal sets," in Proc. IEEE GLOBECOM 2003, San Fransisco, Dec. 1-5,2003, pp. 1978-1983.

[7] P.W. Wolniasky, G.J. Foschini, G.D. Golden, R.A. Valenzua, "V-BLAST: An Architecture for Realizing Very High Data Rates Over the RichScattering Wireless Channel,’ Proceedings IEEE ISSSE-98, Pisa, Italy, 30 Sept. 1998

[8] V. Shashidhar, B. Sundar Rajan and P. Vijay Kumar, "Assymptoticinformation lossless designs and diversity-multiplexing tradeoff," in Proc. IEEE GLOBECOM 2004, Dallas, Nov 29-Dec 3, 2004. 